\title{
How Galileo dropped the ball and Fermat picked it up
}

\author{
Bryan W. Roberts (bwr6@pitt.edu) \\ Department of History and Philosophy of Science \\ University of Pittsburgh
}

\begin{abstract}
This paper introduces a little-known episode in the history of physics, in which a mathematical proof by Pierre Fermat vindicated Galileo's characterization of freefall. The first part of the paper reviews the historical context leading up to Fermat's proof. The second part illustrates how a physical and a mathematical insight enabled Fermat's result, and that a simple modification would satisfy any of Fermat's critics. The result is an illustration of how a purely theoretical argument can settle an apparently empirical debate.
\end{abstract}

Keywords: foundations of physics, history of mathematics, freefall, acceleration, Galileo, Fermat 


\section{Introduction}

The modern textbook concept of uniform acceleration is given as a definition: it is a constant change in velocity over time. This definition is in accord with experience; for example, it correctly describes objects falling freely near the surface of the earth. However, the nature of uniform acceleration was once a mammoth foundational problem. At the birth of modern mechanics, there was neither an obvious definition nor a body of experimental data to characterize the concept. Nevertheless, the problem turned out have a theoretical solution, through a proof that one side of the debate was in contradiction with a well-known empirical fact. That proof is the subject this paper.

One part was easy. Namely, it was clear that uniform acceleration is characterized by a constant change in velocity. The challenge was in answering the question, change with respect to what: distance, or time? The correct answer is time. But why isn't it distance? And can a convincing argument be established in the absence of empirical data?

The seeds of an argument were suggested in a notoriously terse passage of Galileo's Two New Sciences. However, as modern scholars have pointed out ${ }^{1}$, the first convincing argument was actually given by Pierre Fermat, of 'Fermat's last theorem' fame. Scholars have announced scattered conclusions about Fermat's analysis. Galluzzi calls Fermat's proof "rigorously geometrical," and a "deduction of the Galilean proportion of the acceleration of natural motion" (Galluzzi, 2001, 258). Drake has said that there is "no getting around Fermat's argument" (Drake, 1989, 75). However, these conclusions have not only been superficial; they have often been incorrect. Fermat's proof is not geometrical; it does not deduce any Galilean proportion; and there do seem to be clear ways to get around Fermat's argument.

I would like to correct the story of the Galileo-Fermat analysis of acceleration. This story reveals several surprising results about how a concept of empirical significance, which lies at the heart of a physi-

${ }^{1}$ See especially (Mahoney, 1973, 384-387), but also (Drake, 1975a, 360), (Drake, 1975b, 45), and (Drake, 1989, 75). 
cal theory, can be settled through theoretical analysis alone. The four central results that I will argue for are the following:

1 The physical insight behind the analysis of acceleration was that the well-known law of constant velocity actually places restrictions on motion that is not constant.

2 The mathematical insight behind the analysis was that if velocity increases in discrete jumps, then as we scale those jumps down, the result becomes more and more like a continually increasing quantity.

3 There is an objectionable part to the Galileo-Fermat analysis, from the point of view of a critic that would reject any argument involving infinite sequences.

4 Nevertheless, a slight modification of the argument appears capable of satisfying any such critic.

The result was a theoretical argument, showing that the traditional empirical characterization of freefall was in contradiction with the law of uniform velocity.

The paper has two main sections. The first section sketches the background of the analysis, by recounting some key events leading up to Fermat's proof. Here I briefly sketch the debate between two schools of freefall, as well as Galileo's argument in response to this debate. In the second section, I'll describe the analysis that Fermat presents. In particular, I'll draw out a physical and a mathematical insight, and show how they enabled Fermat's argument. Finally, I'll point out some possible objections that Fermat's contemporaries might have given, and illustrate a simple modification that addresses these concerns.

\section{How Galileo Dropped the Ball}

Let's begin with a little narrative. There were two competing schools of freefall in the years leading up to Fermat's analysis: a Galilean school and a Jesuit school. The views of these schools were logically incompatible, and a curious dispute arose between them. This dispute was exacerbated by a cryptic argument of Galileo, which suggested that the controversy could be settled by purely theoretical considerations. However, Galileo's argument was generally thought to be incomplete and unconvincing.

The following four subsections fill in the details of this story. The goal is to understand how this foundational issue arose, as a debate over 
two empirical characterizations of freefall. We will then be prepared to see Fermat's proof, which shows how one of these characterizations contradicts a well-known fact.

\subsection{Two Schools of Freefall}

The death of Galileo in 1642 marked the birth of a vigorous debate over the nature of freefall. The traditional position was upheld most loudly by French Jesuits. They stated that the velocity of an object in freefall is proportional to the distance fallen. More precisely, their claim was that:

$$
\frac{v_{2}}{v_{1}}=\frac{d_{2}}{d_{1}}
$$

where $d_{1}, d_{2}$ are any two distances below the point from which an object is released, and $v_{1}, v_{2}$ are the velocities of the object at those positions $^{2}$. For ease of reference, let's call Equation (1) the Jesuit law of freefall.

In contrast, the Galilean law of freefall maintained that velocity in freefall is proportional to time fallen:

$$
\frac{v_{2}}{v_{1}}=\frac{t_{2}}{t_{1}}
$$

where $t_{1}, t_{2}$ are any two times after the object is released, and $v_{1}, v_{2}$ are the respective velocities at those times.

The Galilean claim is equivalent to the time-squared law ${ }^{3}$. So, Galileo's commitment to Equation (2) was already implicit in the Dialogue of 1632, where he famously wrote that in freefall, "the spaces passed over are to each other as the squares of the times" (Galilei, 1967, 222). But the time-squared law apparently did not generate much controversy until 1638, when it made an explicit appearance as a logical consequence of the Galilean law $(2)^{4}$. A lively debate then ensued, and lasted for nearly a decade.

2 Throughout this paper, I use equality of fractions as shorthand for the language of compound ratios. In particular, a sentence like ' $A$ is to $B$ as $C$ is to $D$ ' will be simply written $A / B=C / D$. If there is any question, the reader can always substitute the original language.

3 There is an easy proof of this in modern language: let $s$ be the distance traveled, $t$ the time passed, and $v$ the velocity of a body dropped from rest at $t=0$. If $s \propto t^{2}$, then $v=d s / d t \propto 2 t \propto t$. Conversely, if $v \propto t$, then $s=\int_{0}^{t} t d t=t^{2} / 2 \propto t^{2}$.

4 See (Drake, 1989, chapter 7) for a discussion. 


\subsection{InCOMPATiBility of The Two Schools}

Marin Mersenne, a heavy-weight of French intelligentsia, applauded Galileo's time-squared law in his 1639 notes on the Two New Sciences. He also picked up on a now-notorious suggestion of Galileo: that if the Jesuit law were true, then motion would be instantaneous. Quite a damning conclusion. But Mersenne didn't buy it, suggesting that the Jesuit law "can nevertheless be understood in a correct way; for can we not say that the speed is greater in proportion to the greater spaces traversed?" 5 .

Mersenne had nailed precisely the part Galileo's remark that seems to lack justification (more on this in Section 2.4). However, Mersenne's comment also betrays some confusion. Once one has accepted the Galilean law of freefall, there is no 'correct way' to understand the Jesuit law, because these two laws are incompatible. One proof of this is the following. Suppose, for reductio, that both (1) $v_{2} / v_{1}=d_{2} / d_{1}$, and (2) $v_{2} / v_{1}=t_{2} / t_{1}$. Then:

$$
\begin{aligned}
\frac{t_{2}}{t_{1}} & =\frac{d_{2}}{d_{1}} & & \text { by substituting (1) into (2) } \\
& =\frac{\left(t_{2}\right)^{2}}{\left(t_{1}\right)^{2}} & & \text { by substituting the time-squared law. }
\end{aligned}
$$

Simplifying this equation, we get the strange result that

$$
t_{2}=t_{1}
$$

In other words, not rejecting either (1) or (2) would entail that any two distances traveled in freefall are traversed in the same amount of time. But that's obviously false: drop any object, and observe that it reaches your knees before it reaches your ankles. So, by reductio, a view that supports (1) is incompatible with a view that supports (2).

This argument is just one of innumerable other ways of illustrating this incompatibility. Of course, Mersenne was not a mathematician, and so his apparent failure to deduce this result is perhaps not surprising. But a more adept theoretician could have easily reached this conclusion. And that might explain some of the vigor with which the participants in this debate went after each other. We will now discuss one particularly vigorous such exchange, which directly inspired Fermat's work.

\footnotetext{
5 "neantmoins cecy se peut entendre d'une veritable façon; car pourquoy ne peuton pas dire que la vitesse est plus grande à proportion des plus grands espaces parcourus?" (Mersenne, 1973a, 184).
} 


\subsection{Une Bataille de Pierres}

By the 1640's, the disagreement between the Galilean and the Jesuit schools had developed into a lengthy published debate. Among the most hard-headed debaters were Pierre Gassendi (who supported the Galilean school of freefall), and Pierre Cazré (who upheld the Jesuit school). From 1642 to 1646, these two Pierres kept a long written correspondence of courteous defamation. It unfolded roughly as follows.

Gassendi (1642) set out to show, among many other things, that the Galilean law of freefall was a consequence of the impellent force of air, combined with the attractive force of the earth. Cazré (1645a) responded to Gassendi with a scathing criticism of the Galilean school. In this letter, Cazré described a balance experiment with which he claimed to have verified the Jesuit law. Gassendi (1646) shot back with a meticulous response to Cazré's critique, and Cazré (1645b) replied with a counter-argument ${ }^{6}$. And the exchange could have continued, had Gassendi not withdrawn.

Part of the curiosity of the episode is that, although one might have expected a simple experiment to provide quick resolution of the controversy, such an experiment was not soon to arrive. Both Gassendi and Cazré described experiments that they claimed would allow anyone to verify their respective views. Each decried that the other's experiments as fallacious. So, little progress was made by either Gassendi or Cazré on the empirical front.

On the other hand, the problem was an excellent candidate for pure theoretical resolution. Both Gassendi and Cazré agreed about one substantive physical fact: if the velocity of a body is uniform, then

$$
\frac{v_{2}}{v_{1}}=\frac{d_{2}}{d_{1}} \frac{t_{1}}{t_{2}} .
$$

This law is easy to verify experimentally. Perhaps that's why Gassendi and Cazré managed to agree. Perhaps it was simply because this law was as old as the Physics of Aristotle, or because it enjoyed the irrefutable status of what Lakatos called the 'hard core' of a research program. Either way: the uniform velocity law provided a hopeful starting point for the theoretician. Any argument that assumed nothing more controversial than Equation (3) would be sure to convince members of both schools of freefall. In what follows, we will see that this is exactly the rhetorical strategy that Fermat pursued.

${ }^{6}$ This debate has been discussed in detail by numerous authors; see (Clark, 1963), (Drake, 1975b), (Galluzzi, 2001), (Palmerino, 2002), (Palmerino, 2004). 


\subsection{Galileo's 'Very Clear Proof'}

Could the Jesuit law be disproven, without assuming the Galilean law? Could it be disproven without assuming anything more controversial than the uniform velocity law? (Take a moment to try this before you read on - it's easier said than done!) Galileo seemed to think so, and provided a notorious sketch of a proof in the Two New Sciences:

Sagredo: That the falling heavy body acquires force in going, the speed increasing in the ratio of the space... appear to me as propositions to be granted without repugnance or controversy.

Salviati: And yet they are as false and impossible as that motion should be made instantaneously, and here is a very clear proof of it. When the speeds have the same ratio as the spaces passed or to be passed, those spaces come to be passed in equal times; if therefore the speeds with which the falling body passed the space of four braccia were the doubles of the speeds with which it passed the first two braccia, as one space is double the other space, then the times of those passages are equal; but for the same movable body to pass the four braccia and the two in the same time cannot take place except in instantaneous motion. (Galilei, 1974, 160.)

Salviati's response to Sagredo would appear to stretch the meaning of 'very clear proof.' The second part is not so bad: if all spaces really were passed in equal times during freefall, then one might be able to conclude that freefall would be instantaneous (more on this in Section 3 ). But why should we believe that, if the Jesuit law of freefall is true, then all spaces are passed in equal times in freefall? Galileo leaves this problem to his readers.

Galileo's readers did not generally find an answer forthcoming. Marin Mersenne raised early doubts, as we have discussed above. But many modern commentators have also found themselves at a loss. Indeed, some have suggested that Salviati's terseness is simply a "trait de plume," or even that Galileo was committing the novice error of "applying the law of uniform motion to a motion which is not so"7.

The most compelling of all such speculations was given around 1646 , with the entrance of a third Pierre into the debate. Pierre Fermat agreed that Galileo's conclusion remained "undemonstrated," but suggested that Galileo might actually have had a correct proof in mind $^{8}$. Fermat wrote down what he thought Galileo meant in a lengthy letter to

\footnotetext{
7 "Précisons que l'erreur de Galilée consiste à appliquer la règle des mouvements uniformes à un mouvement qui ne l'est pas" (Mersenne, 1973b, 250).

8 Sed concedatur, si placet, viro perspicaci et Lyncaeo indemonstrata conclusio, dummodo sit vera (Fermat, 1894, 268).
} 
Gassendi. At the end of the letter, Fermat implored Gassendi: "dismiss any troubles from Cazré or any other adversary of Galileo, as well as the innumerable volumes that might crop up, which by a single demonstration will be either refuted or proven useless and superfluous, by the confessions of the authors themselves" ". It was a bold suggestion. In the next section, we'll get to the bottom of Fermat's argument.

\section{How Fermat Picked The Ball Up}

Let's begin with a wide-angle overview of the proof. I'll then follow through on my promise, to show how Fermat's result hinges on a physical insight and a mathematical insight. Next, I'll run through a step-by-step review of Fermat's proof, in order to understand the machinery of how it works. Finally, we'll turn to some of the objections that Fermat's contemporaries might have given.

\subsection{Fermat's Proof: A Wide Angle View}

In a debate between two incompatible empirical laws, Fermat proposed a theoretical resolution. How can such an argument be carried out? One way to do it is to show that one of the two empirical laws contradicts something that everybody accepts. This was the core of Fermat's argument: although it's not obvious, the Jesuit law of freefall actually contradicts the law of constant velocity ${ }^{10}$. Here's a wide-angle view of how the argument runs.

Fermat's proof has a two-step structure, which superficially follows Galileo's 'very clear proof.' The first step shows that the Jesuits are committed to the claim that a falling body travels different distances in the same amount of time. The second step argues that as a consequence, motion in freefall occurs instantaneously. Like Galileo, Fermat takes this result to be absurd. So, he concludes by reductio that the Jesuit law of freefall is false.

Both of Fermat's two main insights went into the first part of the proof, and it's the trickiest part of the argument. Fermat began with an arbitrary geometric sequence of segments ${ }^{11}$, drawn along the path traveled by a falling body (Figure 1a below). He then considered time required for a freely falling body to traverse any two consecutive lengths

\footnotetext{
9 "ne tibi in posterum facessat negotiuim aut Cazraeus aut quivis alius Galilei adversarius, et in immensum excrescant volumina, quae unica demonstratione, vel fatentibus ipsis auctoribus, aut destruentur aut inutilia et superflua efficientur" (Fermat, 1894, 276).

10 That is, that velocity is proportional to distance/time.

11 A geometric sequence is one in which the ratio of successive terms is constant.
} 
in the sequence, say $A B$ and $B C$. Fermat was then able to prove the following: "The time for the accelerated motion through $A B$ is therefore not less than the time for the accelerated motion through $B C$; but neither is it greater...; therefore it is equal" ${ }^{12}$. In short: a freely falling body traverses each segment in the sequence in the same amount of time.

The second part of the proof was comparatively easy. There, Fermat showed that the first step implies instantaneous motion. To see why, he simply noted that a finite interval can contain as many segments of a (converging) geometric sequence as you want. So, if the time required to traverse each of these segments is finite and non-zero, then a freely falling object will require an arbitrarily long amount of time to traverse the entire finite interval. Fermat thus concluded that the interval is traversed in no time at all.

In sum: Fermat showed that the Jesuit law of fall implies an absurd conclusion, that motion in freefall can occur instantaneously.

Mahoney has given a lovely overview of Fermat's steps, pointing out that the proof "rests on the clever use of continued proportion" (Mahoney, 1973, 387). But one would like to dig more deeply into the machinery of Fermat's proof. What was Fermat's physical insight? And, if 'continued proportions' were his main mathematical tool, then how did he use them?

Fermat's physical insight was to use facts about uniform velocity to place upper and lower bounds on the velocity of a falling body. I call this insight physical, because it's the only step in the argument that makes use of an empirical assumption: the law of uniform velocity. Fermat's mathematical insight was, as Mahoney suggests, to make use of continued proportions, known today as geometric sequences. In particular, Fermat used facts about these sequences to 'tighten' his upper and lower bounds on the velocity of a falling body. Indeed, the tighter these bounds were made, the closer they got to approximating the continuous acceleration of a falling body. The details of these techniques are the subject of the next two subsections.

\subsection{Physical Insight}

Fermat's central physical insight is easy to overlook, buried deep in lengthy mathematical prose. Even worse, Fermat introduces the upper and lower bounds at different points in the proof, and does so with very

\footnotetext{
12 Non ergo tempus motus accelerati per $A B$ est minus tempore motus accelerati per $B C$; sed nec majus, ut supra demonstratum est : ergo est aequale (Fermat, 1894, 274).
} 


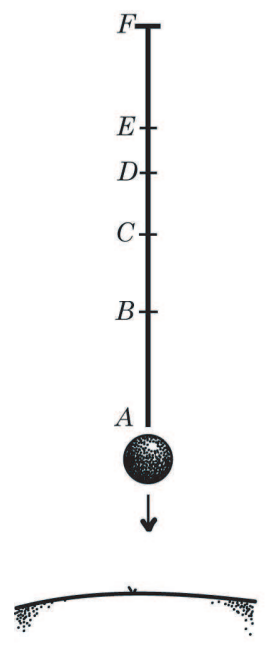

(a)

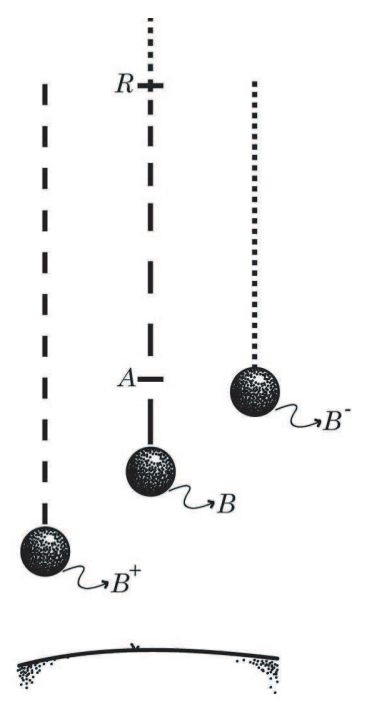

(b)

Figure 1. (a) A geometric sequence $F A, F B, F C, \ldots$, set out along the path of a falling body. (b) Three bodies in motion: $B$ is in freefall, while $B^{+}$and $B^{-}$move with constant velocity $v^{+}$and $v^{-}$, respectively.

little discussion. So, let's begin with a clearer illustration of the basic idea.

Fermat considers a vertically standing segment $A R$ of length $d$, with $R$ toward the top. Suppose that a body $B$ is dropped somewhere above $A R$, accelerates downward in freefall, and traverses $A R$ in a certain time $t$. Let's say that $B$ has initial velocity $v^{-}$when it gets to $R$. We'll say it has final velocity $v^{+}$by the time it gets down to $A$.

Next, Fermat asks us to consider the "imaginary motions" 13 of two more bodies, $B^{-}$and $B^{+}$. Neither of these two bodies are in freefall. Instead, Fermat takes that initial velocity $v^{-}$, and imagines the body $B^{-}$moving with constant velocity $v^{-}$across $A R$. Let's say that $B^{-}$ traverses $A R$ in time $t^{-}$. Similarly, Fermat takes the final velocity $v^{+}$, and imagines the body $B^{+}$moving with uniform velocity $v^{+}$. Let's say it traverses $A R$ in time $t^{+}$. The situation is illustrated in Figure $1 \mathrm{~b}$ above.

The interesting fact about this construction, Fermat says, is that "the time $[t]$ for the accelerated motion through $A R$ is less than the time $\left[t^{-}\right]$for the uniform motion through $A R$ with the velocity at $R$

13 motus fictitii (Fermat, 1894, 272). 
$\left[v^{-}\right]^{\prime 14}$. This provides him with an upper bound on the time $t$ for the accelerated body $B$ to cross the segment. Similarly, he concludes that the time for the accelerated motion "is greater than" 15 the time $t^{+}$. This provides him with a lower bound. In summary:

$$
t^{+} \leq t \leq t^{-}
$$

This simple insight is the key to Fermat's result. He has in effect taken a quantity that people disagreed about (time elapsed in accelerated freefall), and bounded it using quantities that everyone agreed about (time elapsed in uniform motion). Notably, this strategy is much in the spirit of the Archimedean double-reductio, which often sought to calculate controversial quantities like areas under curves, by bounding them with more tractable shapes like rectangles (Archimedes, 1912, e.g., 251-252).

Let's now take this a step further. The bounds stated in Equation (4) certainly make intuitive sense. But suppose an objector were to challenge these inequalities. Could any further justification be given? Curiously, Fermat suggests the further justification is that "by the hypothesis, the velocity would continually increase from $R$ to $A$ "16. What does Fermat mean here?

I suggest we adopt two interpretive views of Fermat's claim. First, let's assume Fermat is using "the hypothesis" in the same way that he uses it throughout proof: to refer to the reductio hypothesis, the Jesuit account of freefall. Second, let's take the phrase "continually increase" [crescat] to mean increase whenever distance and time increase. (This is similar to what we now call a monotonic increase). These two interpretations are certainly plausible. But they are also fruitful, in that they allow us take Fermat's suggestion seriously, and deduce Equation (4) from the Jesuit law of freefall. This derivation can be carried out in two steps.

First, let's see how Fermat can get 'continually increase' from the Jesuit law. We can take the 'increasing' part for granted, since no one believed that an object slows down once released into freefall. More interestingly, observe that the Jesuit law of freefall

$$
\frac{v_{2}}{v_{1}}=\frac{d_{2}}{d_{1}}
$$

\footnotetext{
14 Sed tempus motus accelerati per $A R$ est minus tempore motus per $A R$ uniformis juxta velocitatem in $R$ (Fermat, 1894, 271).

15 est majus (Fermat, 1894, 274).

16 cum enim a puncto $R$ usque ad punctum $A$ perpetuo, ex hypothesi, velocitas crescat (Fermat, 1894, 271).
} 
implies immediately that if $d_{1}<d_{2}$, then $v_{1}<v_{2}$. So the Jesuit law does indeed come with an assumption of monotonicity or 'continual increase' built in.
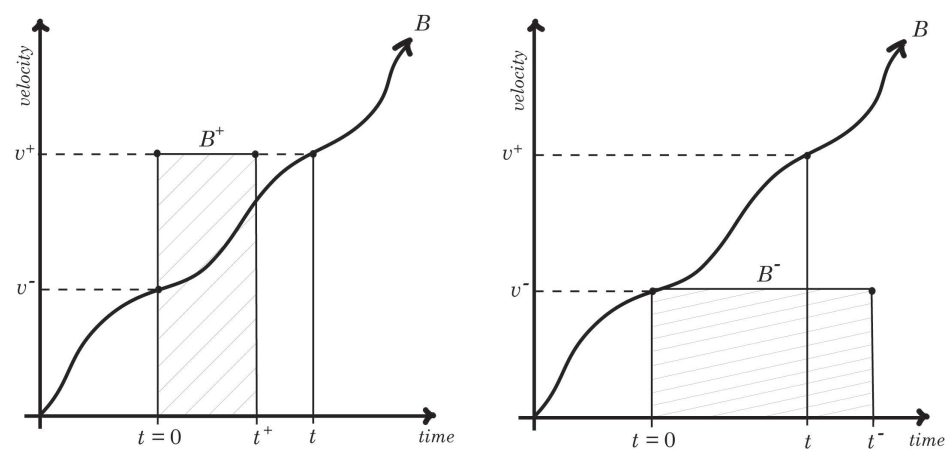

Figure 2. If the velocity of $B$ increases monotonically, then the requirement that the areas under these three curves be equal guarantees that $t^{+}<t<t^{-}$.

Next, consider what a 'continual increase' means in the example of three bodies in motion $\left(B, B^{+}\right.$and $\left.B^{-}\right)$. All three of these bodies are imagined to traverse a particular, vertically oriented segment of length $d$. They arrive at the top of the segment at the same time (let's say $\left.t_{0}=0\right)$, and proceed to move down it. In particular, $B, B^{+}$and $B^{-}$ traverse the segment in times $t, t^{+}$and $t^{-}$, respectively. Their motions are illustrated in Figure 2.

Now, the path of the falling body $B$ can be drawn almost arbitrarily, since we know little about how its velocity is changing. However, we do know that its velocity will 'continually increase' over distance and time. However, each of the three bodies travel the same distance $d$. So, no matter how much time each one requires to make the trip, they will all have the same area under the curve (as illustrated). Given this constraint, it's easy to deduce the bounds given by Equation 4 from Figure 2. If $t^{+}$were any greater than $t$, then the area under $B^{+}$would be greater than the area under $B$. That's impossible, so $t^{+} \leq t$. Similarly, $t^{-}<t$ is only possible if the area under $B$ is greater than the area under $B^{-}$. So it must be that $t^{-}>t$.

These graphs offer a convenient way to illustrate how the bounds in Equation (4) follow from the Jesuit law, as Fermat suggested. However, one must remember that this is almost certainly not the way that Fermat made the argument. (For example, it is unlikely that he thought of distance as area under a curve.) On the other hand, one can check that an isomorphic argument is available using only simple 
algebra $^{17}$. So although Fermat left us sparingly little to work with, this reconstruction may offer a glimpse into the kind of argument he had in mind.

Fermat's physical insight, in summary, is that bodies moving with constant velocity can provide upper and lower bounds on the time that elapses during accelerated freefall. These bounds make intuitive sense, but they also follow directly from the Jesuit law (and, incidentally, from the Galilean law as well). So the force of this insight would have been felt by both schools of freefall.

Of course, to get a more precise fix on the time $t$ of the freely falling body, one would like to squeeze these upper and lower bounds as close together as possible. Fermat chose to do this using a mathematical construction of which he was a master: the method of constant proportions. Fermat's use of this construction is the subject of the next section.

\subsection{Mathematical Insight}

Recall again the Jesuit law of freefall: for a falling body that begins at rest,

$$
\frac{v_{2}}{v_{1}}=\frac{d_{2}}{d_{1}}
$$

This claim is formulated in terms of ratios. So, in order to apply Fermat's upper and lower bounds on time in freefall, the bounds constructed in the discussion above must also be formulated as ratios. This is achieved by simple division. If $t_{1}$ and $t_{2}$ are the times a falling body takes to travel two respective distances $d_{1}$ and $d_{2}$, then it follows from Equation (4) that

$$
\frac{t_{2}^{+}}{t_{1}^{-}} \leq \frac{t_{2}}{t_{1}} \leq \frac{t_{2}^{-}}{t_{1}^{+}} .
$$

By the uniform velocity law, this in turn implies that

$$
\frac{d_{2}}{d_{1}} \frac{v_{1}^{-}}{v_{2}^{+}} \leq \frac{t_{2}}{t_{1}} \leq \frac{d_{2}}{d_{1}} \frac{v_{1}^{+}}{v_{2}^{-}} .
$$

But there is a problem. Equation (5) is supposed to hold for a falling body that begins at rest, $v^{-}=0$ (remember, $v^{-}$is the minimum velocity of the falling body on a given interval). In this case, (6) implies the trivial result that

$$
0 \leq \frac{t_{2}}{t_{1}} \leq \infty
$$

\footnotetext{
17 Define $\bar{v}:=d / t$, the average velocity of the falling body on the interval. Since $v$ is continually increasing, $v^{-} \leq \bar{v} \leq v^{+}$. Therefore, $d / t^{-} \leq d / t \leq d / t^{+}$, which implies that $t^{+} \leq t \leq t^{-}$.
} 
These bounds are not very informative; they are in a sense 'infinitely wide.' Fermat's strategy is thus to 'tighten' them as much as possible, through the clever manipulation of geometric sequences. Here is the mathematical trick that is the basis for this strategy.

Consider a body that falls from rest at a point $F$, down to a point $A$. Divide this interval $F A$ up into segments by marking off the points $B$ through $E$, such that the sequence

$$
F A, F B, F C, \ldots
$$

is set out in constant proportion. Following (Euclid, 1956)[books XIIIIX], a sequence is in constant proportion if the ratio of any two consecutive terms in the sequence is fixed (i.e., the sequence is geometric). Fermat draws particular attention to one property that the elements of such a sequence share: "their differences will be in the same ratio" 18 . What he means is that for any two terms in the sequence, say $F A$ and $F B$,

$$
\frac{F A}{F B}=\frac{F A-F B}{F B-F C}=\frac{B A}{C B}
$$

Fermat states this claim without argument, but it has a simple proof $^{19}$. This property is evidently important to Fermat, as it is the only mathematical fact that he sets apart as a separate proposition in his letter. Indeed, it's really the mathematical heart of Fermat's proof. The fact allows Fermat to construct a new, finer geometric sequence,

$$
B A, C B, D C, \ldots,
$$

which by Equation (9) is in the same constant proportion as the original sequence. The construction is illustrated in Figure 3. Here are the two reasons it's so important in Fermat's argument.

First, a falling body that begins at $F$ has non-zero velocity on every segment of this construction. So, since $v^{-} \neq 0$ on the segments, we'll get finite (instead of 'infinitely wide') bounds on the time it takes a body to fall across them. Indeed, as we'll see in the next subsection, if we imagine a body with constant velocity that increases in discrete 'jumps,' then we can get bounds on this time that are as tight as we want.

\footnotetext{
18 earum intervalla erunt in eadem ratione (Fermat, 1894, 268).

19 Suppose $a_{1} / b_{1}=a_{2} / b_{2}=\cdots=a_{n} / b_{n}$. Then for any $i, a_{n} b_{i}=b_{n} a_{i}$. Summing over $i$, it follows that

$$
n b_{n}\left(a_{1}+a_{2}+\cdots+a_{n}\right)=n a_{n}\left(b_{1}+b_{2}+\cdots+b_{n}\right) .
$$
}

Therefore, for any $i$,

$$
\frac{a_{1}+a_{2}+\cdots+a_{n}}{b_{1}+b_{2}+\cdots+b_{n}}=\frac{a_{n}}{b_{n}}=\frac{a_{i}}{b_{i}} .
$$


Second, this new construction is nothing more than a scaled-down copy of the original ${ }^{20}$. It is the combination of this fact with with the Jesuit law of freefall that is the central step in Fermat's reductio. Fermat calls his construction as a "sub-proportion" 21 . But for our purposes, it is more appropriate to call it the scaled-down sequence with respect to the original. The significance of this is that we can substitute the ratio of two segments of a geometric series interchangeably with the segments of a scaled-down sequence, since scaled-down sequences preserve ratios of terms.

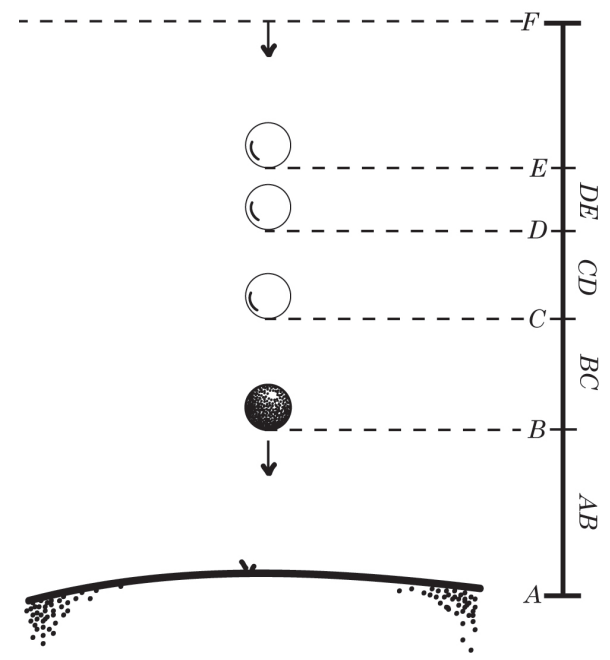

Figure 3. A geometric sequence $F A, F B, F C, \ldots$, has a corresponding 'scaled-down sequence' $A B, B C, C D, \ldots$.

To recap: in the last subsection, I claimed that Fermat's physical insight was to set particular bounds on the time it takes a freely falling body to traverse an interval. I've now suggested that his mathematical insight was to construct a scaled-down sequence along that interval, in order to tighten the bounds. It's now time to see precisely how these insights work together in Fermat's proof.

\subsection{The First Step: Equal Times}

Fermat begins by asking us to imagine a body released at a point $F$, which accelerates in freefall down to a point $A$. As described above, Fermat constructs a sequence $F A, F B, F C, \ldots$ in constant proportion

\footnotetext{
${ }^{20}$ Just take any geometric sequence $\left\langle a k^{i}\right\rangle$; Fermat's new sequence $\left\langle a k^{i}-a k^{i-1}\right\rangle$ $=(1-1 / k)\left\langle a k^{i}\right\rangle$ is just the original sequence scaled by a factor of $(1-1 / k)$.

${ }^{21}$ proportio sub (Fermat, 1894, 270).
} 
along the path of this falling body. He states his goal: "We will demonstrate first that the spaces $C B, B A$ are traversed in the same time, given the supposition [i.e., the Jesuit law] about their motion" ${ }^{22}$. In other words, Fermat will deduce in this first step that the Jesuit law implies that

$$
\frac{t_{B A}}{t_{C B}}=1 .
$$

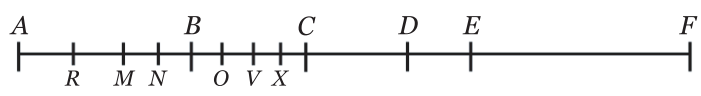

Figure 4. A body falls from $F$ to $A$. The segments $A R, R M, M N, \ldots$ form a geometric sequence, of which $A B, B C, C D, \ldots$ is a subsequence.

This step makes use of an Archimedean double-reductio. Fermat first shows that $t_{B A} / t_{C B} \ngtr 1$, and then shows that $t_{A B} / t_{B C} \nless 1$. Since both halves of the reductio follow exactly the same steps, let's just review the first half.

Suppose, for the purposes of reductio, that $t_{B A} / t_{C B}>1$. Fermat begins by embedding the original sequence $F A, F B, F C \ldots$, in a new sequence $F A, F R, F M, \ldots$, as shown in Figure 4 . Call the ratio of consecutive terms in the new sequence $\rho(=F A / F R)$. Then since $\rho$ can be made arbitrarily close to 1 , the sequence can always be constructed so as to have the property that

$$
1<\rho<\frac{t_{B A}}{t_{C B}}
$$

Fermat remarks, "Who doesn't see that this will indeed occur out of necessity, even from a single term, this operation having been iterated as often as need be?" 23 - but he does not prove the claim ${ }^{24}$.

That's the basic construction. Now, here's the trick. We've constructed a new sequence of segments, with the ratio of consecutive segments $\rho<t_{B A} / t_{C B}$. But as we discussed above, Fermat had the

\footnotetext{
${ }^{22}$ Demonstrabimus primo spatia $C B, B A$ eodem tempore in supposito motu percurri (Fermat, 1894, 269).

${ }^{23}$ quod quidem necessario eventurum, vel ex sola mediae inventione ejusque iterata, quoties opus fuerit, operatione, quis non videt? (Fermat, 1894, 270).

${ }^{24}$ However, Fermat's remark does suggest a simple proof. We must show that any geometric sequence $\left\langle a k^{i}\right\rangle$ can be embedded in a finer sequence $\left\langle a h^{i}\right\rangle$, with the ratio of consecutive terms $\rho=a h^{n+1} / a h^{n}=|h|$ as close to 1 as we like. But $\left\langle a k^{i}\right\rangle$ can be embedded in any sequence $\left\langle a h^{i}\right\rangle$, as long as $h=k^{1 / n}$. So we can just check, for a given $n$, if $|h|$ is small enough. If it's not, then we can increase $n$, which has the effect make $h$ closer to 1 . Therefore, by iterating this procedure 'as often as need be,' we can make $|h|$ be as close to 1 as we like, and thus less than $t_{B A} / t_{C B}$.
} 
physical insight to see that the law of uniform velocity restricts the fraction on the right. In particular, we know that

$$
\frac{t_{B A}}{t_{C B}}<\frac{t_{B A}^{-}}{t_{C B}^{+}}
$$

Fermat's trick is to show that, if the Jesuit law is true, then the right side of the inequality is less than $\rho$. This would imply the left side of the inequality is also less than $\rho$. And that contradicts Equation (11), completing the reductio.

So, how does Fermat get this result out of the Jesuit law? Recall our notation from the previous sections: $v_{I}^{-}$and $v_{I}^{+}$are the minimum and maximum velocities of a falling body on an interval $I$. And $t_{I}^{-}$and $t_{I}^{+}$ are the times that would be required for bodies traveling with uniform velocity $v_{I}^{-}$and $v_{I}^{+}$to traverse the interval. Then Fermat's argument can be summarized:

$$
\begin{array}{rlrl}
\frac{t_{R A}^{-}}{t_{O B}^{+}} & =\frac{R A}{O B} \frac{v_{O B}^{+}}{v_{R A}^{-}} & & \text {(by the uniform velocity law) } \\
& =\frac{R A}{O B} \frac{v_{B}}{v_{R}} & \text { (by definition of } \left.v^{+} \text {and } v^{-}\right) \\
& =\frac{F A}{F B} \frac{v_{B}}{v_{R}} & \text { (substituting the 'scaled-down' sequence) } \\
& =\frac{F A}{F B} \frac{F B}{F R} & \text { (by the Jesuit law of freefall) } \\
& =\frac{F A}{F R}=\rho & \text { (by the definition of } \rho) .
\end{array}
$$

But then, as Fermat observes, "the time for the accelerated motion through $A R$ is less than the time $\left[t_{R A}^{-}\right]$for the uniform motion through $A R$ with the velocity at $R\left[v_{R A}^{-}\right]^{\prime 25}$. A similar upper bound can be placed on the time for the accelerated motion through each of the other intervals. Therefore, Fermat concludes,

$$
\frac{t_{B A}}{t_{C B}}<\frac{t_{R A}^{-}+\cdots+t_{B N}^{-}}{t_{O B}^{+}+\cdots+t_{C X}^{+}} .
$$

In effect, Fermat has shown how uniform acceleration across an interval is bounded from above, by uniform motion that increases in discrete 'jumps' across each of the intervals $R A, M R \ldots$. It follows immediately that since each $t^{-} / t^{+}$is equal to $\rho$,

$$
\frac{t_{R A}^{-}+\cdots+t_{B N}^{-}}{t_{O B}^{+}+\cdots+t_{C X}^{+}}=\rho .
$$

\footnotetext{
${ }^{25}$ Sed tempus motus accelerati per $A R$ est minus tempore motus per $A R$ uniformis juxta velocitatem in $R$ (Fermat, 1894, 271).
} 
But, combining this result with Equation (13), we have now shown that

$$
\frac{t_{B A}}{t_{C B}} \leq \rho .
$$

That contradicts Equation (11). So by reductio, $t_{B A} / t_{C B} \ngtr 1$.

Exactly the same kind of argument can be used to complete the other horn of the reductio, and show that $t_{B A} / t_{C B} \nless 1$. Thus, we have by double-reductio that $t_{B A}=t_{C B}$. Indeed, this argument can be made for any pair of consecutive intervals in the sequence, $B A, C B, D C, \ldots$. So, Fermat concludes, as a body falls freely from $F$ to $A$, the Jesuit law implies that "each and every one of the spaces is traversed in the same time" 26 .

\subsection{The Second Step: Instantaneous Motion}

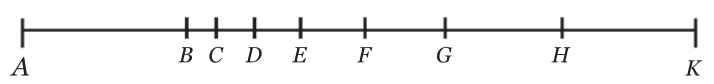

Figure 5. A body at rest at $A$ falls down to a point $K$. The sequence $K H, H G, G F$ is a geometric sequence that converges before getting to $A$.

Fermat's second step is comparatively easy. Here, one simply shows that the above conclusion leads to an absurdity: that motion in freefall is instantaneous. Fermat begins by imagining a body falling from rest at a point $A$ down to a point $H$, and then continuing further down to a point $K$ (Figure 5). We are instructed to assume, for the purposes of reductio, that this motion "does not in fact occur instantaneously," but that it "will require a certain definite time" 27 . Then there is some integer $n$ such that

$$
(n) t_{H K}>t_{A H}
$$

As before, Fermat now constructs a geometric sequence in which the first term is $K H$, the second term is $H G$, and so on. But this time, he must suppose in addition that the first $n+1$ segments of the series $H G+G F+F E+\cdots$ sum to less than the length of $H A$ (where Fermat has assumed the fact discovered by Archimedes that a geometric series can converge). Now, by the first step of Fermat's proof, the falling body takes the same amount of time to traverse these $n+1$ of these segments. So if $H B$ is the union of the $n+1$ segments, then the time it takes the falling body to traverse all of them is

$$
t_{H B}=(n+1) t_{H K} .
$$

\footnotetext{
26 omnia omnino spatia eodem tempore percurri (Fermat, 1894, 275).

$27 \mathrm{Si}$ enim motus per $H K$ non fiat in instanti, fiet in tempore aliquo determinato (Fermat, 1894, 275).
} 
Substituting equation (15) into (14), we now get that

$$
\frac{n}{n+1} t_{H B}>t_{H A} \Rightarrow t_{H B}>t_{H A} \text {. }
$$

Fermat thus writes that, "a fortiori, the time of the motion through $H B$ is greater than the time of the motion through all of $H A$, which is absurd" 28 . Having produced an absurdity, Fermat now finally rejects the reductio hypothesis, and infers that the Jesuit law implies that freefall occurs instantaneously. He concludes: "Thus, the assertion of Galileo is true, even though he did not himself demonstrate it" 29 .

\subsection{OBJECTIONS}

Drake wrote that "There is no getting around Fermat's argument in support of Galileo's position" (Drake, 1989, 75). That is certainly correct from a modern perspective, since we now know that Galileo was right and the Jesuits were wrong. But from a more historically sensitive perspective, Drake's conclusion here is a bit too hasty.

Fermat deduced an absurdity, as Galileo indicated was possible, by assuming (i) the Jesuit law of freefall; (ii) the law of uniform velocity; and (iii) the validity of a set of mathematical techniques. By reductio, one of these assumptions must be rejected. Fermat chose to reject the Jesuit law. But an objector might just as well reject one of his other assumptions, and thus 'get around' Fermat's purported vindication of the Galilean school. But would any of Galileo's contemporaries have been willing to reject one of these assumptions? I will argue in defense of Drake, that although there are ways to get around Fermat's result, none of them are reasonable options for any of Fermat's contemporaries.

The law of uniform velocity was simply too well established in Fermat's time to have been objectionable to anyone. So the only real target available to an objector was option (iii), Fermat's mathematical techniques. Of course, most of these techniques were Euclidean and uncontroversial. But Fermat might have heard some complaints about what appears to be 'infinitary reasoning' in his argument. In the 17th century, the many methods of 'indivisibles' and of 'infinitesimals' were only just beginning to be understood. They were often viewed with distrust, as these methods appeared to be wrought with paradoxes. Galileo himself, even in his later work, urged caution in considerations of the infinite, when "paradoxes are at hand" (Galilei, 1974, 28). These

\footnotetext{
28 ergo a fortiori tempus motus per $H B$ tempore motus per totam $H A$ est majus. Quod est absurdum (Fermat, 1894, 275).

29 Ergo vera remanet Galilei illatio quamvis eam ipse non demonstrarit (Fermat, 1894, 276).
} 
were matters of serious concern to potential objectors, and so there are at least two parts of Fermat's proof that might have been deemed worrisome. The first worry, I think, is a clear red herring; it only superficially appears to deal with the infinite. The second worry is less superficial; however, I argue that an easy modification of Fermat's argument would have been accepted even by mathematical conservatives.

The first worry comes in Fermat's conclusion to his first step. Here, Fermat writes,

the time for the accelerated motion through $C D$ is equal to the time for the accelerated motion through $A B$, and to the time for the accelerated motion through $B C$, and continuing the reasoning, if desired, to the infinite, each and every [omnia omino] one of the spaces is traversed in the same time ${ }^{30}$.

While the language 'omnia omnino' might have sent up warning flags for some critics of 'infinite quantities,' Fermat never actually uses this claim about the infinite in any quantitative way. Recall that this claim only gets used in the second step, when Fermat picks out $n+1$ segments in a geometric sequence (where $n$ is finite), and concludes that they are all traversed in the same amount of time. Thus, although Fermat's language here is suggestive of infinity, his reasoning is not.

The second worry is somewhat more interesting. This is about the assumption that a geometric series can converge. Fermat assumes here that, given any finite interval $A K$, a geometric sequence exists that begins with a particular segment $H K$, and can be continued through arbitrarily many terms without every reaching $A$ (as in Figure 5 above). Fermat does not explain why this assumption should be true. So it appears that an objector is free to complain that Fermat is making illegitimate use of infinitary reasoning: he has claimed that a particular, infinite geometric sequence can occupy a finite length.

However, there are two responses available to Fermat. First, infinite geometric sequences would hardly have constituted 'fringe' calculations using infinity. Indeed, the notion of a convergent geometric series played a central role in Archimedes's well-known letter on the quadrature of the parabola (Archimedes, 1912, Proposition 23-24). So this objection would perhaps only have held sway among extreme mathematical conservatives.

But in fact, Fermat's position was even stronger. Even if Fermat grants this worry, there appears to be an easy modification of Fermat's proof that avoids this 'infinitary assumption' altogether. Go back to

\footnotetext{
30 Eadem ratione patet tempus motus accelerati per CD aequari tempori motus accelerati per $\mathrm{AB}$, et tempori motus accelerati per $\mathrm{BC}$, et, continuatis, si placet, in infinitum rationibus, omnia omnino spatia eodem tempore percurri (Fermat, 1894, $275)$.
} 
end of the first step, in which Fermat showed that a falling body takes the same amount of time to traverse any two lengths in a geometric sequence. One immediate consequence is that any two lengths at all are traversed in the same amount of time in freefall.

To see why, let $a_{1}$ be an arbitrary length, which is the first element in some geometric sequence. Any two segments in this sequence are traversed in the same amount of time, according to Fermat's first step. Moreover, all the elements are determined as soon as we choose $a_{2}$. But $a_{2}$ can be any arbitrary length. Therefore, any arbitrary length is traversed in the same amount of time as $a_{1}$. So, since $a_{1}$ was also chosen arbitrarily, any two lengths are traversed by a falling body in the same amount of time.

Now, in particular, we can conclude that any two halves of a unit segment are traversed in the same amount of time as the segment itself. Therefore, $2 t=t$, a contradiction. This argument is obviously much easier than the one that Fermat actually gives in his second step. Furthermore, it seems to avoid any possible worries about infinitary reasoning. I can only speculate that Fermat took a more complicated route because he wanted to do more than disprove the Jesuit law: he wanted to prove Galileo's suggestion down to the very letter.

Thus, it seems that Drake was almost correct. There appears to be just one serious objection that could have been leveled against Fermat's construction of a convergent geometric series. But as it turns out, that part of the argument could have been avoided altogether. So the wouldbe objector to Fermat's result would not have gotten very far.

\section{Conclusion}

The debate between the Jesuit and the Galilean schools of freefall was eventually settled empirically, as Newtonian mechanics began to amass evidence at the end of the 17 th century ${ }^{31}$. But remarkably, Fermat managed to produce solid evidence in favor of the Galileans, independently of any empirical discovery, by proving that the Jesuit account was inconsistent. We have now seen that this unlikely result required only a very simple insight into the physics of falling bodies, together with some very clever mathematical techniques. It is only unfortunate that the density of Fermat's argument seems to have prevented it

\footnotetext{
31 It's not clear precisely when the Galilean law of freefall definitively gained widespread acceptance. While still controversial in the 1640 's, it was treated as an established fact in the (1673) Horologium of Huygens. Understanding the evaporation of the conflict over this 30-year period appears to be an open project for investigation.
} 
from drawing much commentary. Gassendi, the original recipient of Fermat's letter, never responded to Fermat's argument in print. Fermat apparently made no efforts to further publicize his work. And so Fermat's contemporaries, as well as modern scholars, seem to have largely ignored this curious little argument.

This kind of historical episode suggests some care be taken in our philosophical accounts of confirmation and theory change. Certainly, a picture in which empirical claims are measured only by their ability problem-solve or determine research agendas would be too blunt ${ }^{32}$. As we have seen, Fermat's refutation of the Jesuit law followed a different strategy. Fermat's proof made a connection that wasn't obvious: that the Jesuit characterization of freefall contradicts the law of uniform velocity. This theoretical solution was enough to settle an empirical debate - a result that I hope both historians and philosophers of science might find of interest.

\section{Acknowledgements}

I would like to thank Paolo Palmieri for many stimulating discussions during the development of this work.

\section{References}

Archimedes: 1912, The Works of Archimedes. New York: Dover.

Cazré, P.: 1645a, Physica demonstratio qua ratio, mensura, modus ac potentia accelerationis motus in naturali descensu gravium determinantur, adversus nuper excogitatam a Galilaeo Galilaei Florentino philosopho ac mathematico de eodem motu pseudoscientiam. Ad clarisimum virum Petrum Gassendum Cathedralis Ecclesiae diniensis praepositum dignissimum. Paris: J. du Brueil.

Cazré, P.: 1645b, Vindiciae demonstrationis physicae de proportione qua gravia decidentia accelerantur. Ad clarissimum D. Petrum Gassendum Cathedralis Ecclesiae diniensis praepositum dignissimum. Paris: G. Leblanc.

Clark, J.: 1963, 'Pierre Gassendi and the Physics of Galileo'. Isis 53, 352-370.

Drake, S.: 1975a, 'Free Fall from Albert of Saxony to Honoré Fabri'. Studies in History and Philosophy of Science 5, 347-366.

Drake, S.: 1975b, 'Impetus Theory Reappraised'. Journal of the History of Ideas 36, 27-46.

Drake, S.: 1989, History of Freefall, Chapt. 7. Toronto: Wall and Emerson, Inc.

Euclid: 1956, The Thirteen Books of the Elements, Vol. 2. New York: Dover Publications, Inc.

\footnotetext{
32 In particular, the Fermat-Galileo episode may require a more refined development of Lakatos' (1968) 'research programmes,' or Laudan's (1981) 'research traditions,' although a more detailed discussion lies outside the scope of this paper. Thanks to an anonymous referee for suggesting this avenue of research.
} 
Fermat, P.: 1894, 'Fermat a Gassendi (1646?)'. In: P. Tannery and C. Henry (eds.): Oeuvres de Fermat, Vol. 2. Paris: Gauthier-Villars et Fils, pp. 267-276.

Galilei, G.: 1967, Dialogue Concerning the Two Chief World Systems. Berkeley and Los Angeles: University of California Press, second revised edition.

Galilei, G.: 1974, Two New Sciences. Madison: University of Wisconsin Press.

Galluzzi, P.: 2001, 'Gassendi and l'Affaire Galilée of the Laws of Motion'. In: J. Renn (ed.): Galileo in Context. New York: Cambridge University Press, pp. 239-275.

Gassendi, P.: 1642, De motu impresso a motore translato: Epistolae duae in quibus aliquot praecipuae, tum de motu universe, tum speciatim de motu terrae attributo, difficultates explicantur. Paris: V. de Lanville.

Gassendi, P.: 1646, De proportione qua gravia decidentia accelerantur: Epistolae tres quibus ad totidem epistolas R. P. Cazraei, Societatis Iesu, respondetur. Paris: L. de Heuqueville.

Huygens, C.: 1673, Horologium oscillatorium sive de motu pendulorum ad horologia aptato demonstrationes geometricae. Paris: Apud F. Muguet.

Lakatos, I.: 1968, 'Criticism and the Methodology of Scientific Research Programmes'. Proceedings of the Aristotelian Society 69, 149-186.

Laudan, L.: 1981, 'A Problem-Solving Approach to Scientific Progress'. In: I. Hacking (ed.): Scientific Revolutions. Oxford: Oxford University Press, Chapt. vii, pp. $144-155$.

Mahoney, M. S.: 1973, The Mathematical Career of Pierre de Fermat, 1601-1655. Princeton: Princeton University Press, 2nd edition.

Mersenne, M.: 1973a, Les nouvelles pensées de Galilée, mathématicien et ingénieur, Vol. 1. Paris: J. Vrin, critical edition.

Mersenne, M.: 1973b, Les nouvelles pensées de Galilée, mathématicien et ingénieur, Vol. 2. Paris: J. Vrin, critical edition.

Palmerino, C. R.: 2002, 'Two Jesuit Responses to Galilei's Science of Motion: Honoré Fabri and Pierre Le Cazre'. In: M. Feingold (ed.): The New Science and Jesuit Science: Seventeenth Century Perspectives, No. 6 in New Studies in the History and Philosophy of Science and Technology. Dordrecht: Kluwer, pp. 187-227.

Palmerino, C. R.: 2004, 'Galileo's Theories of Free Fall and Projectile Motion as Interpreted by Pierre Gassendi'. In: C. R. Palmerino and J. M. M. H. Thijssen (eds.): The Reception of the Galilean Science of Motion in Seventeenth-Century Europe, No. 239 in Boston Studies in the Philosophy of Science. Dordrecht: Kluwer, pp. 137-164. 